\title{
Aproximación conceptual al liderazgo en el ámbito social
}

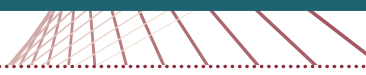

Conceptual approach to leadership in the social realm

\begin{abstract}
Oscar Hernando Cadena Chicunque ${ }^{1}$
oscar.cadena@uniclaretiana.edu.co

Luis Miguel Gallo Díaz'

jefaturatrabajosocial@uniclaretiana.edu.co

Yira Lizet Perea Osorno²

yira.perea@uniclaretiana.edu.co
\end{abstract}

\section{https://doi.org/10.22209/rhs.v7n2a04}

Recibido: julio 26 de 2019.

Aceptado: noviembre 27 de 2019.

\section{Resumen}

El presente artículo da cuenta de los resultados de un proceso de revisión sistemática frente a la categoría liderazgo en el ámbito social. Este proceso se desarrolló desde una perspectiva interdisciplinar en artículos de países iberoamericanos, filtrados bajo criterios de inclusión y exclusión, en bases de datos y motores de búsqueda de libre acceso, entre ellos Redalyc.org y Scielo, elaborados entre los años 2010 y 2017 . En cuanto a los resultados, se identificó que conceptualmente las teorías utilizadas con mayor frecuencia para comprender el liderazgo social son el liderazgo transformacional,

1 Fundación Universitaria Claretiana; Grupo de Investigación Humanidades y Estudios Socioculturales.

2 Fundación Universitaria Claretiana; coordinadora de la Red Colombiana de Semilleros de Investigación nodo Chocó; Grupo de Investigación Humanidades y Estudios Socioculturales. el liderazgo servicial y el liderazgo subjetivo e intersubjetivo. Por otro lado, se evidenció que existen categorías conexas al liderazgo entre las que sobresalen: la comunidad - la sociedad, los compromisos, la ética y moral, la participación social y el empoderamiento. Para finalizar, se aduce que el liderazgo es un concepto relativo en el que, dependiendo del contexto donde se investigue, subyacen características y taxonomías; sin embargo, en el liderazgo social-comunitario se presenta como eje transversal, en la mayoría de conceptualizaciones, el interés genuino por lo colectivo.

Palabras claves: comunidad; empoderamiento; liderazgo; líder comunitario; líder social; sociedad; Trabajo Social.

\section{Abstract}

This paper discusses the findings of a systematic review process carried out regarding the category of leadership in the social realm. This process was developed from an interdisciplinary perspective. A collection of papers from Latin American countries were chosen using the criteria of inclusion and exclusion. These papers were retrieved from open access databases and search engines, including Redalyc.org and Scielo, and were written between 2010 and 2017. The findings show that the most widely used conceptual theories in relation to social leadership are transformational leadership, helpful leadership and subjective and 
intersubjective leadership. Additional categories were found to be related to leadership including: community, society, commitments, ethics and morals, social participation and empowerment. Finally, it is argued that leadership is a relative concept, that is to say, depending on the context in which it is researched, a variety of characteristics and taxonomies underlie. However, regarding the social-community leadership, a genuine interest in the collective is present as a transversal axis in most conceptualizations.

Keywords: community, empowerment, leadership, community leader, social leader, society, social work.

\section{Introducción}

$\mathrm{E}$ presente documento surge del proyecto de Investigación denominado «El liderazgo Social, una potencialidad en los estudiantes de Trabajo Social de UNICLARETIANA», desarrollado por un grupo de docentes investigadores del programa académico mencionado. Este proyecto tuvo como objetivo principal comprender y analizar los aspectos de orden personal y contextual que, desde la perspectiva de una muestra de estudiantes, reconocidos como líderes, pudiesen ser determinantes para entender su condición y rol en la sociedad.

Hablando en específico de la presente revisión sistemática, se tuvo como propósito de conocimiento explorar las diferentes conceptualizaciones del liderazgo social, teniendo como ejes de análisis: nociones, contextos y aspectos metodológicos de diferentes procesos investigativos a nivel nacional e internacional, a fin de reconocer los elementos sustanciales que pudiesen coadyuvar a la comprensión de este fenómeno en el ámbito universitario de los estudiantes del programa de Trabajo Social de Uniclaretiana, tomando como insumo 30 artículos científicos seleccionados aleatoriamente en bases de datos y motores de búsqueda de libre acceso, todo lo anterior desde una perspectiva interdisciplinar.

¿Por qué se hace importante realizar un acercamiento conceptual a una categoría ampliamente investigada? Una respuesta precisa es que, si bien se han construido bastos contenidos investigativos alrededor del liderazgo, estos se asientan en el ámbito empresarial, organizacional y político y no necesariamente en el comunitario; se hace necesario reconocer perspectivas desde lo bibliográfico con el objeto de comprobar o desestimar algunas hipótesis de las que partió el interés de revisión, entre ellas, la afirmación de que el liderazgo en las bases de la sociedad pudiese tener otras condiciones, otros propósitos, otras acciones, sobre todo si lo entendemos como un fenómeno social relativo, el cual tiene unos desempeños y características que posiblemente pudiera nutrir al currículo del programa de Trabajo Social de Uniclaretiana.

Otra de las conjeturas, es que las personas que se consideran como tal (líderes sociales) y que a su vez estudian Trabajo Social, solo pretenden afianzarse teórica, técnica, instrumental y metodológicamente, para potencializar sus habilidades y competencias intrínsecas, desarrolladas tanto subjetiva como intersubjetivamente, en entornos donde funcionan como garantes de los derechos sociales de colectivos o comunidades. 
En consecuencia, como se puede apreciar en la Figura 1, se recurrió a una revisión bibliográfica sistemática, la cual partió de la definición del tema y un objetivo de revisión, se continuó con los criterios de inclusión y exclusión de los artículos, posteriormente con la sistematización de la información y se finalizó con la escritura del documento que implicó la descripción, análisis e interpretación de lo revisado.

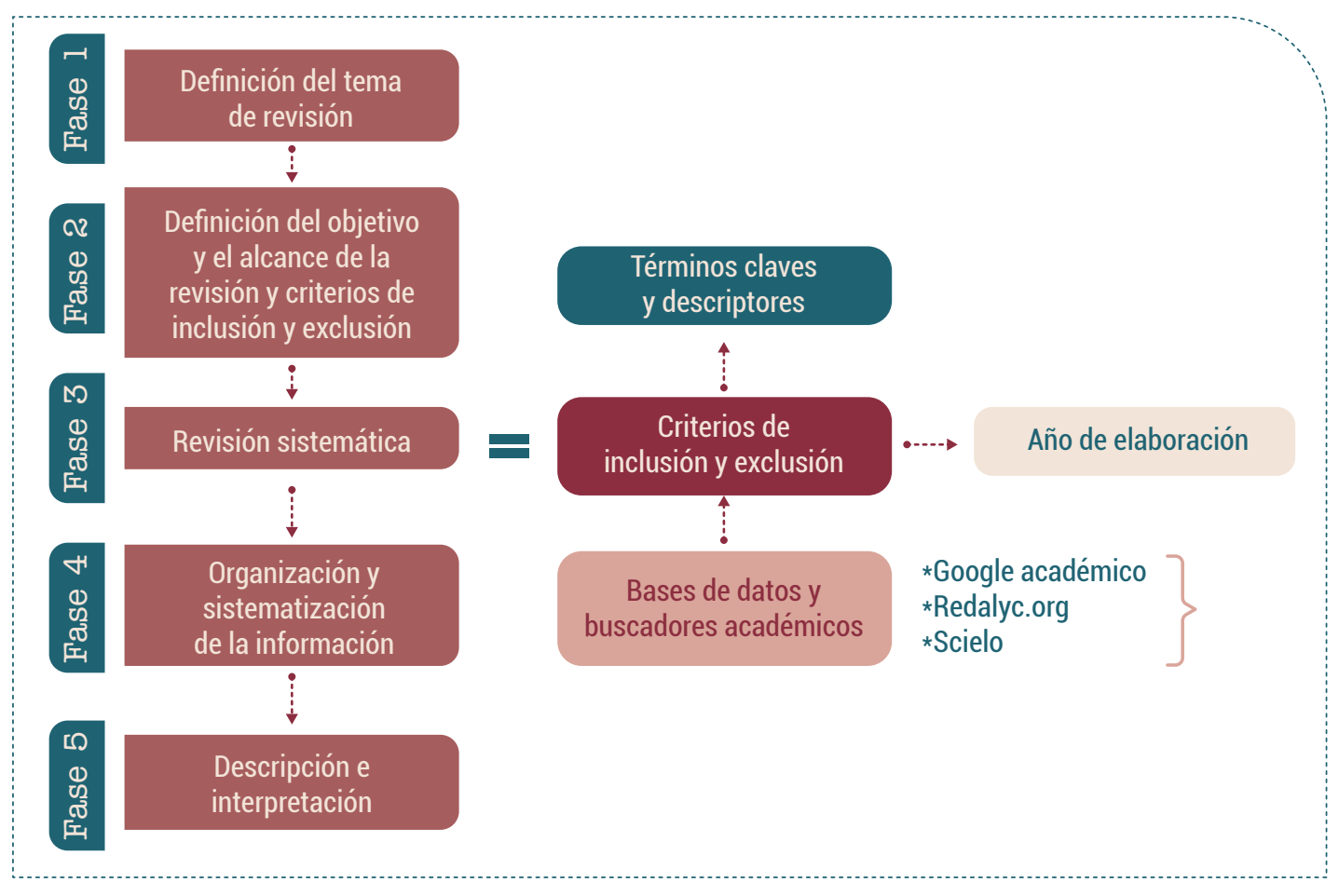

Figura 1. Metodología para la elaboración de artículo.

Fuente: construcción propia.

En esta última etapa del proceso se realizaron las interpretaciones en torno a la categoría eje, conectándolo a contextos como el de educación superior, lo político, escenarios de relaciones horizontales, entre otros; para fines de reconocer aquellas pistas, dimensiones o subcategorías que operacionalizan el liderazgo en el ámbito de lo social, que se convierta en pauta metodológica y, así mismo, sean susceptibles de cotejarse con las fuentes primarias; es decir, aquellas que proceden de la entrevista de la muestra no probabilista del proyecto de investigación y las hipótesis formuladas apriorísticamente.
Respecto a los hallazgos, en términos conceptuales, se identificaron tres perspectivas de análisis e interpretación en los artículos revisados. La primera es la orientación del líder social en el campo político, organizacional, económico-administrativo. Aquí se reconocen teorías clásicas como la de los Grandes hombres o la de los Rasgos. En la misma línea emerge una segunda, un poco más contemporánea, la teoría Transformacional, con gran auge en diferentes escenarios disciplinares, la cual invoca subcategorías como la capacidad que tiene un líder de transfigurar la vida de sus seguidores bajo criterios como la ética y 
la integridad. Finalmente, se identificó una visión emergente que bebe de investigaciones en escenarios como la comunidad, los resguardos indígenas, el barrio, las organizaciones de base, la reivindicación de derechos del territorio y la mujer.

\section{Aspectos metodológicos}

\section{en la revisión sistemática}

Para dar cuenta de este proceso, en primera instancia se definieron tres motores de búsqueda o bases de datos para la revisión del tema delimitado, se identificaron diez artículos en cada una de ellas, es decir, el $33 \%$ de los artículos fueron ubicados en Scielo, otro $33 \%$ en Redalyc.org y, finalmente, se optó por utilizar el motor Google académico con otro $34 \%$. Es relevante mencionar que fue útil añadir al «término clave» o descriptor la expresión «más» la terminación «.PDF», para facilitar la individualización de documentos científicos, tal y como se aprecia en la Figura 2.

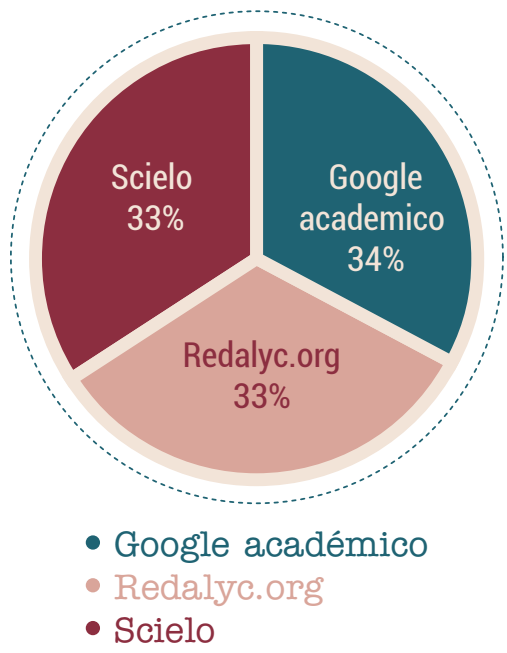

Figura z. Porcentajes artículos según motores de búsqueda.

Fuente: construcción propia.
Hecho el primer filtro, a partir de los motores de búsqueda, se determinaron treinta artículos científicos a los cuales se les realizó una revisión a partir de una matriz que contenía aspectos como: título, autor, año, objetivos, definición de liderazgo, tipificaciones subyacentes desde la perspectiva del autor y un ítem denominado análisis general, según se observa en la Figura 3.

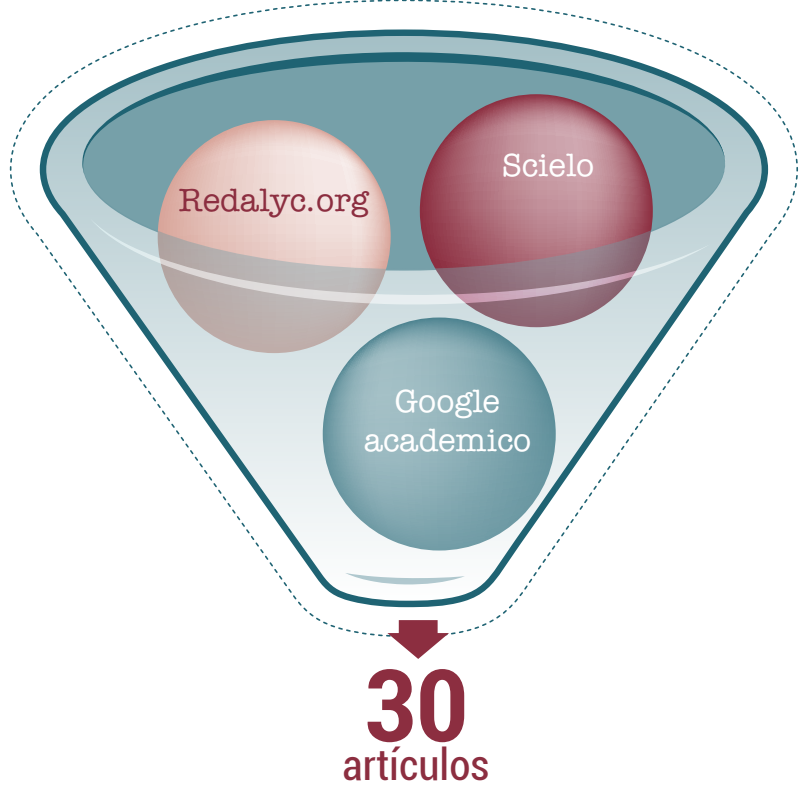

Figura 3. Número de artículos consultados.

Fuente: construcción propia.

Respecto a los términos claves, para realizar la revisión se tuvo en cuenta el enunciado «liderazgo" y se le enlazó palabras como: comunitario, social, político, servicial, empoderamiento, académico; elementos que posteriormente permitieron realizar algunas consideraciones respecto la tipificaciones, taxonomías y formas de análisis de la categoría objeto investigación. Otro aspecto que derivó dentro de este procedimiento fue el surgimiento de otros conceptos que necesariamente fueron claves para el análisis, entre ellos, género, participación, carisma, transformacional. 
En cuanto a los lapsos de tiempo que determinaron la búsqueda, se tuvieron en cuenta dos momentos. El primero comprendido entre los años 2010 al 2012, lapso en el que se ubicaron los documentos científicos escritos por: Alvarado, Moreno, y Rodríguez (2009), Figueroa, y Rodríguez (2012), Labourdette y Scaricabarozzi (2010), Reyes-Jácome y Rodríguez (2011), Soledispa (2012) y Pareja Fernández de la Reguera, López, El Homrani, y Lorenzo Martín (2012).

El segundo incluyó el lapso entre los años: 2013 al 2017, donde se analizaron los artículos desarrollados por: Hernández (2013), Yamila et al. (2015), Hernández, Herrera , y Chávez Mejía (2015), Luque, Rasero, y Arque (2013), Castillo et al. (2017), Zamora (2004), Espinosa y Esguerra (2017), Fernández-Fonseca y Cardona-Sánchez (2017), Revelo y de la Ossa (2014), Fierro y Villalva (2017), Álvarez y González (2014), Castillo, Puigdellivol, y Antunez (2017), Carmona (2017), Álvarez Henao y González Uribe (2014), González, (2014), Velasco (2014), Bustamante y Correa (2017), Erazo, Jiménez, y López (2014), Erazo et al. (2014), Velásquez G., Barreto, y López (2017), Fernández y Cardona (2017), Velásquez G. et al. (2017), Sánchez (2017), Muñoz y Solarte (2015), Mijares (2016), Vázquez, Bernal, y Liesa (2014), Güiza, Rodriguez, Oliva, y Moreno(2016) y Rojas (2013).

\section{Resultados}

\section{De los países donde se originaron los artículos}

En términos del país donde se desarrollaron los artículos revisados, se definieron los siguientes porcentajes (Tabla 1):
Tabla 1. Porcentaje de artículos según países donde se originaron.

\begin{tabular}{|l|l|l|}
\hline \multicolumn{1}{|c|}{ País } & Frecuencia & Porcentaje \\
\hline Argentina & 1 & 3.3 \\
\hline Brasil & 1 & 3.3 \\
\hline Chile & 2 & 6.7 \\
\hline Colombia & 9 & 30.0 \\
\hline Costa Rica & 2 & 6.7 \\
\hline Ecuador & 4 & 13.3 \\
\hline España & 5 & 16.7 \\
\hline México & 3 & 10.0 \\
\hline Venezuela & 2 & 6.7 \\
\hline SL & 1 & 3.3 \\
\hline & & 30 \\
\hline
\end{tabular}

Fuente: Construcción propia.

Como se puede evidenciar en la tabla de frecuencia, el país de donde se inspeccionó el mayor porcentaje de artículos fue Colombia (30\%), prosiguiendo con España y Ecuador, con un $16.7 \%$ y $13.3 \%$ respectivamente y con una menor proporción se reconocieron documentos de países como Chile, México y Venezuela con porcentajes que no superaban el $7 \%$.

\section{De los objetivos}

\section{y propósitos de conocimiento}

Continuando con los elementos generales que caracterizan los artículos, hubo la necesidad de reconocer la orientación o tendencia de los objetivos o propósitos de conocimiento. En este punto se reconoció lo siguiente:

1. Desde resultados de investigación derivados de análisis bibliográficos y aproximaciones conceptuales del liderazgo, se identificaron cuatro artículos de revisión y estados del arte, con enfoques diferentes, aspecto que es particular teniendo en cuenta que el eje de análisis fue el mismo, 
es decir, el vector de búsqueda fue el liderazgo situado en el ámbito social. Es así como Fernández y Cardona (2017) presentan la caracterización de los trabajos investigativos desarrollados alrededor del liderazgo comunitario y liderazgo social, acotados por los grupos de investigación clasificados por Colciencias en Bogotá Colombia, precisando que existe una diferencia sustancial entre los dos conceptos, determinados por la institucionalidad y lo comunitario. En esta misma línea emerge el trabajo escrito por Andrade (2013), en el que se tiene por objetivo presentar otra tipificación del liderazgo comunitario, determinado por la acción colectiva e individual.

Desde un enfoque diferente, Fierro y Villalva (2017) retoman las conceptualizaciones desarrolladas frente el liderazgo participativo, este vinculado a las organizaciones y la teoría de los rasgos, donde se tienen en cuenta nociones como el de liderazgo carismático y autoritario. En este mismo horizonte Espinosa y Esguerra (2017) exponen los aspectos principales que definen y conceptualizan el liderazgo servidor en el escenario organizacional.

De acuerdo con lo anterior, se identificaron dos líneas de análisis en la revisión de aproximaciones conceptuales:

La Figura 4 (parte superior) tiene a su vez dos aristas: el liderazgo social, el cual se relaciona con la racionalidad técnica y especializada conectada con la institucionalidad, y el liderazgo comunitario, concepto de donde derivan dos niveles, el de comunidad y el de por la comunidad, aspectos que se enmarcan en la comprensión del liderazgo en términos de procesos individuales o sinergias colectivas. Sin tratar de llegar a una generalización, se podría deducir que, si bien se hace relación a procesos de revisión sistemática, las orientaciones son disimiles tanto conceptual como contextualmente. La Figura 4 (parte inferior) presenta como eje articulador el contexto de las organizaciones en perspectiva administrativa y la gestión humana, y se conectan dos ópticas del liderazgo, el servicial y el participativo.

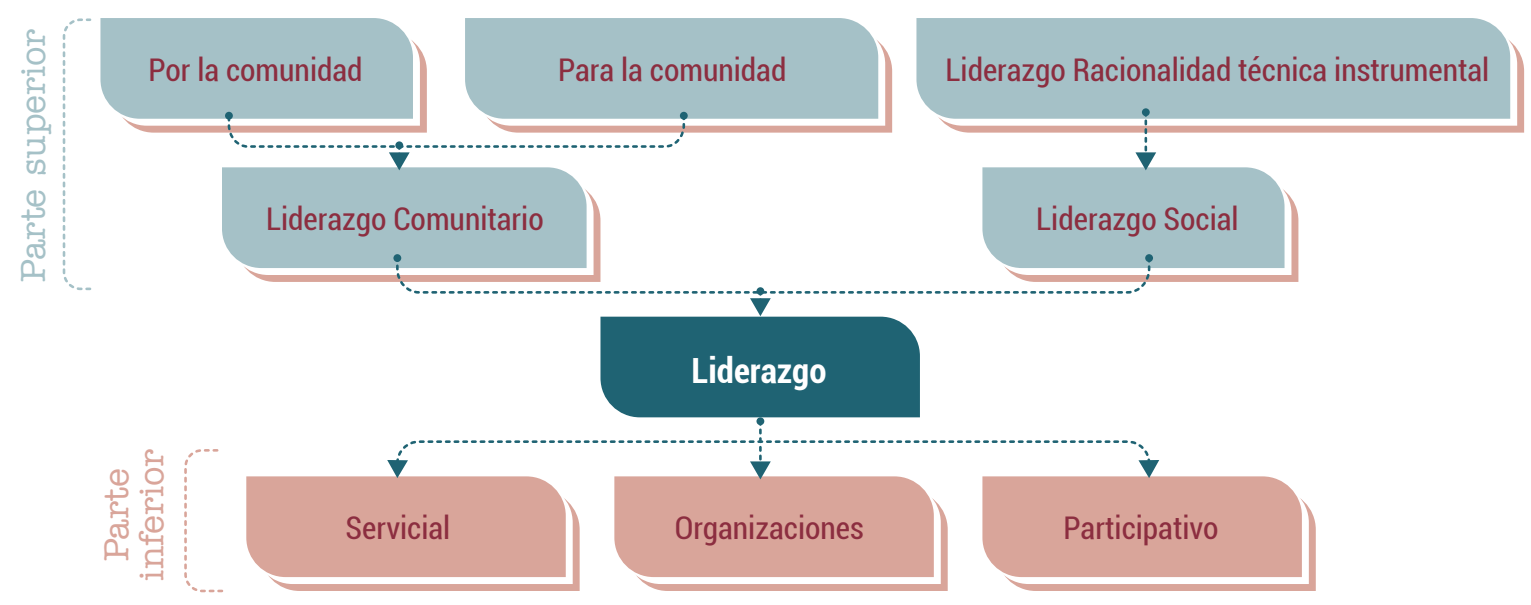

Figura 4. Síntesis de los artículos "Aproximaciones conceptuales y teóricas".

Fuente: construcción propia. 
2. Una tendencia imprevista en los objetivos de los artículos revisados, que si bien no es significativa en términos de frecuencia (10\%), es relevante por el contexto donde se ubica, refiere a procesos de indagación conducentes a la sistematización, comprensión y descripción del liderazgo en el marco de la participación social y procesos de salud pública, ejemplo de ello es lo propuesto por Armijo et al. (2017), Carmona (2017) y Velásquez et al. (2017), indicando categorías conexas como el empoderamiento en comunidades reconocidas por la institucionalidad del Estado desde un enfoque étnico.

3. Por otro lado, se reconoció cierto nivel de afinidad en los artículos resultados de investigaciones en el contexto educativo, en estos, por ejemplo, resulta importante indagar aspectos como: la resignificación del liderazgo académico desde la creatividad, Hernández (2013); el perfil transformacional vinculado al empoderamiento en los procesos académicos en la relación docente-estudiante en el acto educativo, Silva et al. (2015); las relaciones horizontales y decisiones consensuadas desde la guía del liderazgo compartido, Armijo et al. (2017) y Mijares, (2016); la relación cualitativa de la inclusión social, la democracia en la formación del liderazgo en el ámbito educativo para la formación de liderazgos, González (2014) y Vázquez et al. (2014). Cabe mencionar que desde la perspectiva de estas investigaciones el liderazgo se ubica tanto en docentes como en estudiantes.

4. Al continuar con la revisión se reconoció otra tendencia, la del ámbito de lo comunitario y político; muestra de ello, lo expuesto por Hernández et al. (2015), quienes se propusieron investigar algunas organizaciones de México, caracterizando procesos de gestión y capacidades endógenas desde una perspectiva colectiva. Por otro lado, desde el enfoque del empoderamiento y liderazgo femenino, Luque et al., (2013), y Muñoz y Solarte (2015) tuvieron como finalidad analizar y caracterizar las manifestaciones del liderazgo en la mujer desde dos contextos: los inmigrantes africanos y las ONG en el marco de políticas públicas.

5. En el contexto colombiano se identifica otra orientación a lo colectivo, vinculado a dos escenarios, uno el sentido de la eficacia del liderazgo en el contexto de las víctimas del conflicto armado interno del departamento del Atlántico Revelo y de la Ossa (2014) y, el otro, se orienta al análisis de las acciones de participación comunitaria implementadas por voceros de la ruralidad, con el propósito de identificar las potencialidades, dificultades y lecciones aprendidas en torno a la gobernanza ambiental y al empoderamiento comunitario. En este horizonte de comprensión emerge la propuesta de Álvarez y González (2014), quienes se enfocaron desde el ámbito institucional en las dinámicas del presupuesto participativo, tomando como eje dos categorías de reflexión: la participación y el liderazgo comunitario, este último entendido como aquel que emerge desde abajo hacia arriba y media en la relación comunidad-instituciones estatales.

\section{Muestras y contextos}

Respecto a las muestras o actores sociales o participantes objeto de estudio, se caracterizaron los siguientes (Tabla 2): 
Tabla జ. Muestras o actores sociales participantes.

\begin{tabular}{|l|c|c|}
\hline $\begin{array}{c}\text { Muestras 0 } \\
\text { actores sociales } \\
\text { participantes }\end{array}$ & Frecuencia & Porcentaje \\
\hline $\begin{array}{l}\text { Empresarios y trabajadores } \\
\text { en las organizaciones. }\end{array}$ & 3 & $10 \%$ \\
\hline $\begin{array}{l}\text { Docentes investigadores, } \\
\text { estudiantes y directivos en } \\
\text { el ámbito académico. }\end{array}$ & 11 & $37 \%$ \\
\hline $\begin{array}{l}\text { Líderes en el ámbito } \\
\text { comunitario: enfoque } \\
\text { de género, étnico y } \\
\text { reivindicación de derechos. }\end{array}$ & 16 & $53 \%$ \\
\hline
\end{tabular}

Fuente: construcción propia.
El $10 \%$ representa empresarios y trabajadores en organizaciones, porcentaje mínimo acorde con el propósito de la revisión sistemática, el $37 \%$ y el $53 \%$ atañe a docentes, directivos, estudiantes y voceros o procesos comunitarios y políticos, proporciones justificadas en la intención de reconocer aspectos de orden metodológico, teórico y conceptual de procesos investigativos en torno a las categorías liderazgo social y comunitario.

En cuanto a los contextos donde se desarroIlaron las investigaciones (Figura 5), estos son semejantes al de las muestras, es decir, $10 \%$ organizaciones, $37 \%$ ámbito educativo y $53 \%$ el escenario comunitario.

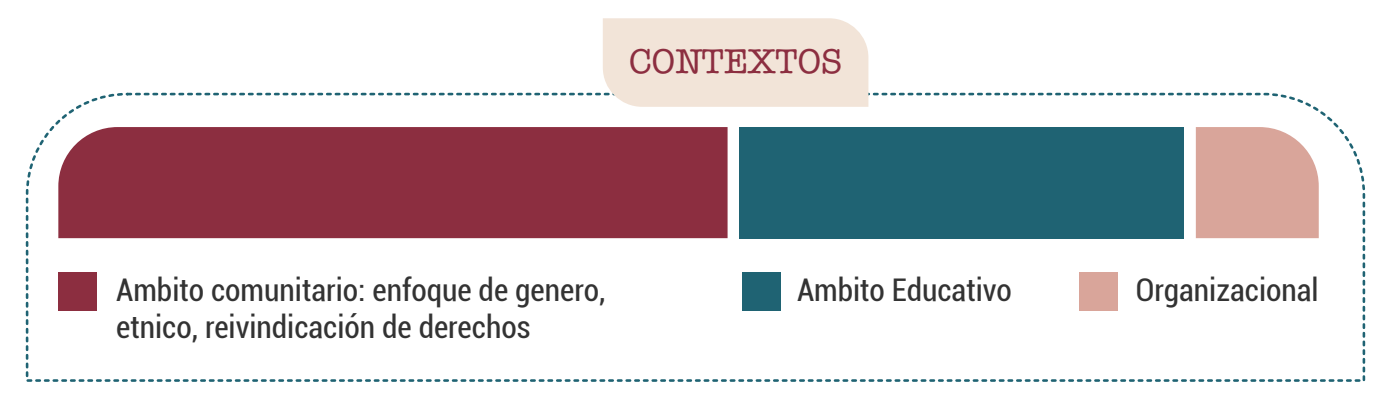

Figura 5. Contextos donde se desarrollaron las investigaciones.

Fuente: construcción propia.

De las teorías que se vinculan al liderazgo en el ámbito social

La categoría del liderazgo ha sido un concepto interpretado desde diferentes perspectivas científicas y disciplinares, entre ellas, la psicología organizacional, la psicología social, la administración de empresas, la economía, las ciencias y estudios políticos, la filosofía, la sociología, la antropología. La pretensión de este documento no es realizar una disertación o análisis exhaustivo de ellas por su amplitud y los contextos en los que se desarrollaron, sin embargo, se postula como pretensión identificar aquellos sistemas teóricos (desde una perspectiva interdisciplinar) que pudiesen dar pistas para acercarse a una conceptualización del liderazgo social o comunitario en el escenario universitario, específicamente en los estudiantes de Trabajo Social de Uniclaretiana. 
Para hacer este rastreo se acudió a las teorías prevalecientes en los artículos revisados. De este ejercicio se denotó lo siguiente. Al tomar como referencia lo expuesto por Fernández y Cardona, (2017) se han estructurado dos paradigmas de comprensión y análisis del liderazgo como fenómeno social. El primero es el denominado como tradicional, es decir, aquel que exalta a los grandes hombres, los iconos históricos que han influenciado política, económica, cultural y socialmente la humanidad, que tienen características intrínsecas desde su nacimiento; aquí convergen teorías como la del liderazgo carismático y parten de fuentes disciplinares como la sociología, la economía y las ciencias políticas. Del mismo modo, en este paradigma emergen propuestas teóricas situacionistas, donde las circunstancias del contexto son determinantes para que aparezca o subyazca el liderazgo. Es preciso mencionar que estas conceptualizaciones han tenido aterrizajes en diferentes escenarios como el de la economía y la política, y ofrecen análisis tanto desde perspectivas neopositivistas como descriptivo-interpretativas, desde otra perspectiva teórica se reconoce un paradigma que parte del análisis de las competencias del líder de potenciar, transformar e inspirar, facilitando problematizaciones de la realidad que impliquen puntos de equilibrio.

En este horizonte es que se identifica que los líderes tienen como rasgo característico el convertirse en "modelos a seguir», aquellos que "comunican una visión clara y un sentido de misión colectiva, muestran comportamientos éticos y morales, se ganan el respeto y la confianza, dan seguridad y son capaces de obtener el esfuerzo extra requerido de lo seguidores» (Silva et al., 2015, p. 73). Del mismo modo buscan potenciar las capacidades de las personas que lideran bajo principios como la solidaridad y un enfoque colectivo, potencialización tal que se instituye a partir de una persuasión entusiasta que reconoce a los individuos y al colectivo al mismo tiempo. Visto desde esta perspectiva, la teoría sugerida es el liderazgo transformacional, propuesta que se sintetiza en los siguientes factores: a) influencia idealizada, b) consideración individualizada, c) estimulación intelectual y d) el liderazgo inspiracional (Bass, 1985).

Desde otra perspectiva, Espinosa y Esguerra (2017) retoman las características de lo que se ha denominado como líder servicial. Este, más que propuesta teórica, se constituye en una postura filosófica que se adentra a los campos de la ética y la moral. Si se pretende comprender epistemológicamente, se considera como un punto de inflexión entre las miradas positivistas e interpretativas y la perspectiva crítico social, teniendo en cuenta que se inscribe a propósitos políticos como la transformación y la emancipación; sin embargo, ha sido estudiado bajo parámetros estandarizados o metodológicos que lo han representado en esquemas y mapas teórico-conceptuales, contextualizados en el ámbito de las organizaciones y las empresas. El autor pionero del líder servicial es Robert K. Greenleaf (2008), quien postuló entre sus características principales la visión, la honestidad, la confianza, el ser ejemplo, tener la apreciación de otros, ser promotor y contar con la habilidad de empoderar a los demás.

Al continuar, desde Mijares (2016), se hace alusión a la noción de liderazgo intersubjetivo y horizontal. Retomando algunas ideas de Freire (1987), explica que el líder debe establecerse en el marco de una relación reciproca, en la que el líder se conecta con las personas que lidera mediante el reconocimiento de sus 
potencialidades creadoras, en ese sentido los dos contribuyen en la emancipación y transformación de su realidad, no se establecen jerarquías en la relación de líderes y liderados. El líder promueve la transformación de aquellos que serán líderes de sus propias vidas individuales y colectivas, en esta mirada persiste un propósito político y emancipatorio que parte del pensamiento crítico, promovido por el líder, a partir de procesos educativos orientados a la toma de consciencia de clase, la cual conlleve a transformaciones de tipo político y económico.

Si bien no se puede postular como un sistema teórico legitimado en comunidades académicas, más bien como una construcción conceptual que se sitúa en los terrenos de la teoría sustantiva y particular del contexto educativo, entendiendo con ello que este concepto no fue configurado a partir de la aplicación de instrumentos Gold standard, por lo contrario, es un conocimiento situado, desarrollado desde la perspectiva interpretativa. Armijo et al. (2017) esclarecen algunos elementos de lo que se denomina como liderazgo compartido y pedagógico, el cual resalta características como: la capacidad de escucha, de diálogo y comunicación, la capacidad de trabajar con grupos, la capacidad reflexiva y autocritica, la capacidad creativa y organizativa, de otro lado se revelan valores y actitudes como: compromiso y automotivación, optimismo, alegría, respeto, tolerancia y solidaridad.

\section{Aproximaciones teóricas} y conceptuales de subcategorías entorno al liderazgo social y comunitario

Uno de los planteamientos con mayor fundamentación es la diferenciación que existe entre la noción de sociedad y la categoría comunidad, disparidad conceptual tal que determina de forma taxativa las distancias que existe entre los intereses que puede albergar un líder en las organizaciones, en las empresas y en lo político, respecto el liderazgo en las organizaciones de base. Se postula que el primero tiene un enfoque más individualista, sustentado en el hombre contemporáneo, competitivo, capaz de liderar, pero bajo intereses propios como la autorrealización académica, profesional o personal. Este tipo de personas tienen un enfoque eminentemente orientado a la productividad utilitaria, de otro lado, emerge aquel líder que tiene visión de colectivo, colectivo entendido como comunidad, aquella que posee un tejido social, que tiene como referencia un territorio, lo cultural y su historia, son capaces de implicarse en procesos bajo principios como la solidaridad y la empatía.

Complementando lo anterior, Fernández y Cardona (2017) postulan, a partir de un estado del arte, dos conceptos que en los imaginarios sociales de la sociedad colombiana se asumen como sinónimos: el liderazgo social y 
el liderazgo comunitario conceptos que pueden reconocerse según el autor como «dos caras de una misma moneda», pero que intrínsecamente sí se distancian. El liderazgo social se imbrica en la lógica de los roles de las empresas y las organizaciones bajo los criterios desempeño y alcance de competencias como:

- Sujetos que sean capaces de orientar y dirigir, con autonomía, engranando esfuerzos para el alcance de metas y objetivos en el ámbito empresarial o sistemas productivo-económicos.

- Pensamiento prospectivo orientado a la producción y a la generación de valor agregado a los productos.

- La capacidad de adaptarse a los desafíos actuales en términos de responsabilidad social y ambiental, con una mentalidad creativa e innovadora.

Con un enfoque diferente el líder comunitario se determina por el contexto, por las luchas sociales en el marco de reivindicaciones colectivas, su identidad se instaura en las dinámicas y propósitos colectivos, posee dominios en lo pragmático y el empoderamiento.

En este punto cabe advertir que estos conceptos no son condicionantes generalizables o definitorios en las capacidades y competencias del líder social y comunitario. Necesariamente, la realidad social por su naturaleza compleja, relativa y contingente impondrá matices grises donde el líder comunitario incorporará a su quehacer aspectos del líder social y viceversa.

Castillo et al. (2017) y Carmona (2017) retoman conceptualmente la categoría de Participación comunitaria como cimiento del liderazgo colectivo; este es entendido como «una labor compartida que tiene diferentes grados de intensidad, durante la cual se generan relaciones de comunicación e intercambio de conocimiento entre las personas, que llevan a procesos de reflexión sobre lo realizado y en consecuencia a cambios de comportamiento» (p. 45). Complementando lo acotado por los investigadores se retoma la propuesta conceptual de Arnstein (1969) respecto de los niveles de involucramiento de la comunidad para determinar su grado de participación; se precisan siete escalones en este proceso: «pasiva, de consulta, de suministro de información, por incentivos, funcional, interactiva y autodesarrollo», así como también el reconocimiento del intercambio de saberes, los consensos y el desarrollo de acciones para el alcance del crecimiento comunitario. Para concluir esta línea, se especifican otros elementos que al parecer son importantes en el marco de la reflexión del liderazgo y la participación ciudadana:

Destacamos que en los procesos de participación dos variables tienen gran importancia, la vocería sin compromiso político y con identificación con su entorno social y el nivel de educación. Cuando esta vocería es asumida por las personas que no tienen compromisos o intereses con grupos políticos, demuestran mayor interés por mejoras en sus comunidades independientemente del apoyo de las autoridades; asimismo, mientras más alto es el grado de educación alcanzado y la identificación del vocero por su entorno, aumenta la necesidad de informarse, capacitarse, organizarse y fortalecer su participación para resolver en colectivo los problemas de su comunidad (Castillo et al., 2017 y Carmona, 2017, p. 22). 
Ahora bien, cuando se menciona el término compromisos, implícitamente se hace referencia a dos escenarios, el primero es el político, el cual se enmarca a intereses individuales y de poder, y el segundo al empeño por la comunidad, aquel que se sustenta por la vocación de servicio:

Un estilo de vida [...] lo cual refiere una combinación entre el compromiso personal y la conciencia social sobre la necesidad de actuar en determinado ámbito de la vida pública. Es un proyecto de vida apoyado por otros bajo una misma identidad, es un desarrollo de acciones que va más allá de una labor remunerada [...] El liderazgo representa la identidad grupal, la expresividad de una comunidad, la socialización e intercambio de experiencias bajo una vocación o incluso un ministerio (Hernández et al., 2015, p. 17).

Adherido a lo anterior, Alvarado et al. (2009) puntualizan otro elemento, se manifiesta que el liderazgo está estrechamente relacionado con la participación comunitaria, en este sentido se aduce que el liderazgo no se le atribuye a una persona, ya que se considera que todos deben aportar al logro de objetivos comunes, por lo tanto, la plataforma del desarrollo comunitario debe ser el empoderamiento de la comunidad, es decir, de sus integrantes y familias, empoderamiento que se entiende como la toma de conciencia del territorio, de su identidad como comunidad y el fortalecimiento de las capacidades propias. En este punto cabe retomar la teoría de la psicología comunitaria de Zimmerman (2000), quien aduce que este proceso parte del reconocimiento del «nosotros» y el sentido de pertenencia para la toma de decisiones que impliquen acciones para un bienestar colectivo.
Para finalizar este aparte, emerge un aspecto crucial, la interacción con el Estado, el cual necesariamente debe ser referente en la comprensión de las dinámicas de participación comunitaria y el liderazgo. Álvarez y González (2014) explican bajo esta mirada que «la participación del líder no es suficiente sin la intervención del Estado» y que el Estado y sus instituciones (idealmente) deben promover acciones tendientes a garantizar la organización comunitaria en procesos participativos y, esto a través de la formulación de proyectos comunitarios que, a la postre, deben aportar nuevas propuestas, sinergia que permitiría que en el interior de las comunidades se establezca un mayor acercamiento entre el Estado y la sociedad, desde la motivación de prácticas participativas, organizadas y reflexivas.

\section{Conclusiones}

Después de realizar la revisión sistemática, a manera de conclusiones se presentan los siguientes aspectos. Respecto a los elementos metodológicos, desarrollar una revisión sistemática implica asociar al proceso de lectura, procedimientos descriptivos e interpretativos que conlleven la configuración de categorías y tendencias que den cuenta de la visión amplia del estado de conocimiento del concepto en términos teleológicos, teórico-conceptuales y metodológicos, complementando lo anterior es imperativo delimitar objetivos de revisión, nivel de profundidad, al tener en cuenta que el conocimiento (en este caso el liderazgo) es basto, situación que si no se tiene límites y puntos de saturación, se vuelve interminable. Del mismo modo, se identificó que Colombia es uno de los países que tiene mayor producción científica de libre acceso respecto el liderazgo 
en el ámbito de lo social. Aspecto que podría denotar que existe un interés manifiesto por la academia de hacer reflexiones frente el papel y rol del líder social en el escenario educativo, político, cultural, ambiental y social, donde emergen conflictos y diferentes tensiones. En esta misma línea, es innecesario caer en el sectarismo epistemológico, metodológico y teórico frente la comprensión y análisis del liderazgo social, por el contrario, se debe tener una mirada compleja de un fenómeno social que es relativo, que condensa una multiplicidad de dimensiones y subdimensiones, que dependiendo del contexto donde se indague subyacen tipificaciones y características.

Dentro de la gama de tipificaciones y taxonomías del concepto de liderazgo que pudiesen engranar al propósito de la revisión sistemática, sobresalen teorías tales como: liderazgo transformacional, el liderazgo participativo, el liderazgo orientado a la emancipación e inclusión social y el liderazgo servicial.

Es necesario mencionar que en el ámbito de lo comunitario el líder social no niega su individualidad o sus intereses propios, pero se diferencia en su propósito trascendental o estructural respecto a otro tipo de liderazgos, es decir, prima la solidaridad, la empatía frente a intereses de orden político y económico, en otros contextos, como en el ámbito académico o de las instituciones educativas, se debe guardar reserva en ubicarlo en alguno de los actores que hacen parte estas dinámicas, al tener en cuenta que este puede estar representado en los estudiantes, en los docentes, o si lo vemos desde la perspectiva «compartida», en toda una comunidad educativa.
Finalmente, en la conceptualización del liderazgo en el ámbito social, además del cuestionamiento respecto a los determinantes que lo configuran en términos subjetivos 0 constructivistas, subyacen otros factores que complejizan su comprensión, por ejemplo, este no necesariamente debe estar representado en un sujeto, puede ser colectivo, en ese sentido surgen conceptos como el de líder y liderazgo; por otro lado, se reconoce a partir del enfoque de propósitos o intereses si este se configura en un liderazgo social o comunitario, o dentro de este último, si es un liderazgo de la comunidad o por la comunidad, determinante que complementa a la perspectiva individual o colectiva del liderazgo.

\section{Referencias}

- Alvarado, A., Moreno, M. E., y Rodríguez, M. C. (2009). Inclusion social y participacion comunitaria: Una perspectiva de trabajo frente a la discapacidad. Ciencia y Enfermeria, 15(1), 61-74. Disponible en: https://doi.org/10.4067/ s0717-95532009000100008

- Álvarez Henao, D., y González Uribe, Y. (2014). Presupuesto participativo, liderazgo comunitario y participación comunitaria: Ejes articuladores para el desarrollo social. Revista Colombiana de Ciencias Sociales, 5(1), 99-121. Disponible en: https://doi.org/10.21501/22161201.963

- Andrade, R. (2013). El liderazgo comunitario y su importancia en la intervención comunitaria. Psicología para América Latina, 57-76.

- Arnstein, Sherry R. (1969). A Ladder of Citizen Participation. Journal of the American Planning Association, 5(4), 216-224. DOI: 10.1080/01944366908977225 
- Bass, Bernard (1985). Leadership and Performance Beyond Expectations. USA: The Free Press.

- Bustamante, M. C. A., y Correa-Chica, A. (2017). Análisis de las variables asociadas al estudio del liderazgo: Una revisión sistemática de la literatura. Universitas Psychologica, 16(1). Disponible en: https://doi.org/10.11144/Javeriana. upsy16-1.avae

- Carmona-Moreno, L. D. (2017). Concepção da Participação social em saúde: Proposta de ressignificação. Revista Ciencias de La Salud, 15(3), 441-454. Disponible en: https:// doi.org/10.12804/revistas.urosario.edu.co/ revsalud/a.6127

- Castillo, A., Corniales, R., Guevara, M., Mazzarri, M., Rattia, J., Karen, F., ... Maruffo, M. (2017). Control De a Edes Aegypti . M Unicipio M Ario B Riceño I Ragorry . V Enezuela ,. Comunidad y Salud, 15(2), 93-101. Disponible en: http://www.redalyc.org/articulo. oa?id=375754623012\%0ACómo

- Castillo, P., Puigdellivol, I., y Antunez, S. (2017). El liderazgo compartido como factor de sostenibilidad del proyecto de comunidades de aprendizajes. Estudios Pedagogicos, 43(1), 41-59. Disponible en: https://doi.org/10.4067/ S0718-07052017000100003

- Erazo, M., Jiménez, M., y López, C. (2014). Empoderamiento y liderazgo femenino; su papel en la autogestión comunitaria en el corregimiento El Hormiguero-Valle Del Cauca. Avances En Psicologia Latinoamericana, 32(1), 149-157. Disponible en: https://doi.org/10.12804/ apl32.1.2014.10

- Espinosa, J. C., y Esguerra, G. A. (2017). Liderazgo servidor, conceptualización, desarrollo e implicaciones para la investigación. Revista Espacios, 38(9). Disponible en: http:// www.revistaespacios.com/a17v38n09/ a17v38n09p21.pdf
- Fernández-Fonseca, E., y Cardona-Sánchez, F. (2017). Los pliegues del liderazgo social y comunitario. Jangwa Pana, 16(2), 197. Disponible en: https://doi.org/10.21676/16574923.2133

- Fernández, P. (2012). El liderazgo en los estudiantes universitarios: una fructífera línea de investigación. Educar, 48, 91-119. Disponible en: https://www.raco.cat/index.php/Educar/article/ view/252994

- Fierro, I., y Villalva, M. (2017). El liderazgo Democrático : Una Aproximación Conceptual. INNOVA, 2(4), 155-162. Disponible en: http:// revistas.uide.edu.ec/index.php/innova/article/ view/210/273

- Figueroa Martha, R. F. (2012). La superación en liderazgo docente y la transformación de los procesos universitarios The bettering process in teaching leadership and the transformation of university processes. Humanidades Médicas, 12(3), 391-408.

- Freire Paulo. (1987). Pedagogía del oprimido (36a. ed.). México: Siglo XXI.

- González, T. (2014). El liderazgo para la justicia social en organizaciones educativas. Revista Internacional de Educación Para La Justicia Social (RIEJS), 3(2), 85-106.

- Greenleaf, R. K. (2008). Who is the servantleader? The Servant As Leader.

- Güiza, L., Rodriguez, D., Oliva, B., y Moreno, S. (2016). Género y empoderamiento comunitario en un contexto de posconflicto: el caso de Vergara, Cundinamarca (Colombia). Estudios SocioJurídicos, 18(2), 115-144. Disponible en: https:// doi.org/10.12804/esj18.02.2016.04

- Hernández, Antonio, J., Herrera, F., y Chávez, C. (2015). Capacidades, liderazgos y estrategias de gestión de organizaciones de la sociedad civil en zonas rurales. Contaduria y Administracion, 60(4), 817-835. Disponible en: https://doi.org/10.1016/j. cya.2015.07.001 
- Hernandez, M. (2013).EL iderazgo académico. Revista de La Educación Superior, 3, 81100.

- Labourdette, S., y Scaricabarozzi, R. (2010). Orientación y Sociedad 2010, N¹0, pp. ISSN 1515-6877. Orientación y Soledad, 10.

- Luque, F. M., Rasero, L. S., y Arque, D. M. (2013). Liderazgo femenino: Un análisis de las diferencias de género en la formación y desarrollo de asociaciones de inmigrantes Africanos. Revista Internacional de Sociologia, 71(EXTRA 1), 141166. Disponible en: https://doi.org/10.3989/ ris.2012.09.24

- Mijares Luna, L. (2016). Liderazgo intersubjetivo: Hacia una emancipación educativa. Ensayos Pedagógicos, 17(1), 73-85.

- Muñoz, S. C. C., y Solarte-Pazos, L. (2015). Empoderamiento de mujeres de una ONG colombiana. Un estudio de caso simple. Revista de Administração Pública, 49(6), 1597-1618.

- Revelo, D. R., y de la Ossa, L. B. (2014). Eficacia de la Participación en organizaciones sociales de víctimas:Percepción de Líderes en El departamento Del atlântico. Analisis Politico, 27(82), 72-87. Disponible en: https://doi. org/10.15446/anpol.v27n82.49408

- Reyes-Jácome, L., y Rodríguez, G. L. (2011). El liderazgo integral en las organizaciones. Avances En Psicologia Latinoamericana, 29(1), 148-160.

- Rojas, R. (2013). El liderazgo comunitario y su importancia en la intervención comunitaria. Psicología Para América Latina, 25, 57-76.

- Sánchez, A. (2017). Empoderamiento, liberación y desarrollo humano. Psychosocial Intervention, 26(3), 155-163. Disponible en: https://doi. org/10.1016/j.psi.2017.05.001

- Silva, F., Gandoy, F., Jara, C., y Pacenza, I. (2015). Liderazgo del docente y niveles de empoderamiento de los estudiantes en un seminario de prácticas comunitarias de una universidad pública argentina. Cuadernos de Administración, 37(54), 68-79.
- Soledispa, M. L. F. (2012). Principales modelos de liderazgo: su significación en el ámbito universitario. Humanidades Médicas, 12(3), 515-530. Disponible en: http://www. humanidadesmedicas.sld.cu/index.php/hm/ article/view/252/167

- Vázquez, S., Bernal, J., y Liesa, M. (2014). La conceptualización del liderazgo: una aproximación desde la práctica educativa. REICE: Revista Electrónica Iberoamericana Sobre Calidad, Eficacia y Cambio En Educación, 12(5), 79-97.

- Velasco Ortiz, L. (2014). Organización y liderazgo de migrantes indígenas en México y Estados Unidos. El caso del FIOB. Migración y Desarrollo, 12(23), 97-125. Disponible en: https://doi. org/10.35533/myd.1223.Ivo

- Velásquez G., V. F., Barreto, Y. M., y López, A. L. (2017). Empoderamiento de líderes comunitarias afrocolombianas desde la Atención Primaria de Salud. Avances en Enfermería, 35(2), 131145. Disponible en: https://doi.org/10.15446/ av.enferm.v35n2.54986

- Zamora, M. (12 de noviembre de 2015). Liderazgo, Derechos Humanos y Seguridad Ciudadana. Ponencia: Liderazgo, Derechos Humanos y Seguridad Ciudadana. S.L, Costa Rica: Acta Academica.

- Zimmerman, M. (2000). Empowerment theory. En J. Rappaport y E. Seidman (Eds). Handbook of community psychology (pp. 43-63). New York, NY: Kluwer. 